\title{
2- 羟基吡啶质子转移过程的理论研究*
}

\author{
陈文凯许娇章永凡周立新李俊馢
}

(福州大学化学系, 结构化学国家重点实验室, 福州 350002)

\begin{abstract}
摘要 采用量子化学中的密度泛函理论, 在 B3LYP/6-31G (d) 基组水平上, 计算并考察了 2 - 羟基吡啶分子醇式 结构和酮式结构进行结构互变的质子转移过程中的 4 种可能途径: (a) 分子内质子转移, (b) 水助催化质子转移, (c) 同种二聚体双质子转移和 (d) 异种二聚体间双质子转移. 计算结果表明, 途经 $\mathrm{c}$ 所需要的活化能最小 (2.6 $\mathrm{kJ} \cdot \mathrm{mol}^{-1}$, 逆反应则为 $\left.27.1 \mathrm{~kJ} \cdot \mathrm{mol}^{-1}\right)$, 而过程 $\mathrm{a}$ 所需要的活化能最大 $\left(137.2 \mathrm{~kJ} \cdot \mathrm{mol}^{-1}\right)$, 途径 $\mathrm{b}$ 和 $\mathrm{d}$ 的活化 能居中间 (分别为 38.7 和 $17.3 \mathrm{~kJ} \cdot \mathrm{mol}^{-1}$ ). 研究还表明, 氢键在降低反应活化能方面起着重要的作用.
\end{abstract}

关键词：2- 差基吡啶，密度泛函理论，量子化学，互变异构体，活化能 中图分类号： 0641

2- 差基吡啶存在 2 种互变异构体 (见图 1). 在 这 2 种异构体之间, 可以通过质子的转移发生结构 互变, 这一反应被认为是杂环化合物中的羰基 - 差 基互变的典型 ${ }^{[1]}$. 由于质子转移而产生的互变异构 现象, 不但在一般化学研究中会发生 ${ }^{[2 \sim 5]}$, 在分子生 物学上也同样具有非常重要的意义, 例如在一些核 酸碱基和很多生物酶催化反应过程中也存在类似的 作用和反应 ${ }^{[6 \sim 11]}$. 已经有很多有关这两种异构体物 理化学性质的实验研究和理论计算, 也有研究异构 体之间的平衡和溶剂效应的半经验和从头算的报 导 ${ }^{[12]}$. 本文采用量子化学中的密度泛函理论, 在 B $3 L Y P / 6-31 G(d)$ 水平上, 找到了 2 条比文献报导 的水催化结构互变反应所需活化能更低, 因此也更<smiles></smiles>

图 12 - 羟基吡啶的 2 种互变异构体

Fig. 1 Two tautomers : 2-hydroxy pyridine and $2(1 \mathrm{H})$-pyridone

2001-12-07 收到初稿, 2002-05-06 收到修改稿. 联系人: 李俊 福建省青年科技人才创新项目资金 (2001J018)资助项目
容易发生的反应路径.

\section{1 计算原理和方法}

差基吡啶的酮式结构和醇式结构 (分别以 Py 和 Hy 表示)进行结构互变时, 设想可以通过如图 2 所示的 4 种反应途径进行. 途径 $\mathrm{a}$ 为通过分子内质 子转移反应直接进行; 途径 $\mathrm{b}$ 为以水为催化剂进行 的异构反应; 途径 $\mathrm{c}$ 是 2 个羟基吡啶分子结合生成 二聚体 $\mathrm{Hy} \cdots \mathrm{Hy}$ 复合物后进行; 途径 $\mathrm{d}$ 是羰基结构 和羟基结构结合生成混合二聚体 Hy $\cdots P y$ 复合物后 进行的异构反应. 其中后 3 种途径均为进行 2 个质 子同时转移的异构反应. 途径 $\mathrm{c}$ 和 $\mathrm{d}$ 末见文献报导.

本文采用密度泛函理论中的 B3LYP 方法 ${ }^{[13,14]}$ (使用含有极化函数的 6-31G(d) 基组水平),计算得 到了全部反应物 (复合物), 产物 (复合物) 和过渡态 的优化几何构型 (采用 Berny 法 ${ }^{[15]}$ 优化). 文献报 导, STO-3G 基组对每一个 $p$ 轨道仅有一个收缩基 函数, 对于计算含氢键的键长效果不好, 同时若没有 在氮原子上加人 $d$ 轨道极化函数, 则分子倾向于平 面构型, 因此采用较大的、同时加人 $d$ 轨道极化函 数是必要的, 才有可能获得合理的分子结构 ${ }^{[3]}$. 同 时进行了零点振动能 (ZPVE) 的校正; 最后还进行了 
<smiles>C#CC#COc1ccccn1</smiles>

Hy<smiles>C#CC#C</smiles>

TS1<smiles>O=c1cccc[nH]1</smiles>

Py

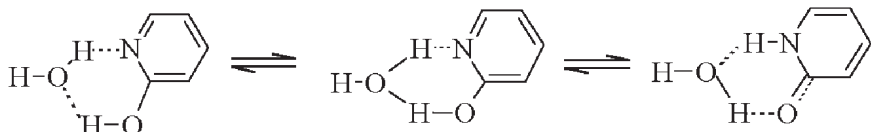

$\mathrm{Hy} \cdots \mathrm{H}_{2} \mathrm{O}$ IM1

TS2

Py $\cdots \mathrm{H}_{2} \mathrm{O}$

b

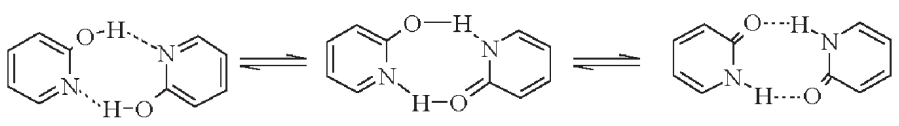

Hy $\cdots$ Hy

IM3

TS3

Py $\cdots$ Py

IM4

c

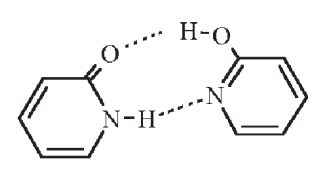

Py $\cdots$ Hy

IM5<smiles></smiles>

TS4

d

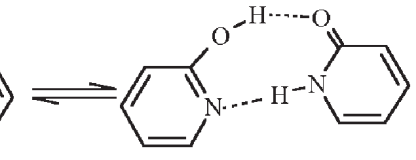

Hy. P Py

IM6

图 2 2- 羟基吡啶进行结构互变的 4 种可能途径

Fig. 2 Schematic drawings of four possible reaction pathways for proton transfer in 2-hydroxy pyridine

分子振动频率分析, 对过渡态进行了确认．全部计 算工作使用 Gaussian 98W 软件包 ${ }^{[16]}$, 在 Intel Celeron 850MHz 计算机上完成.

\section{2 结果与讨论}

在 B3LYP/6-31G $(\mathrm{d})$ 水平上, 计算得到了 4 种 互变过程中各个物种的平衡几何参数, 限于篇幅, 仅 将部分与质子转移过程密切相关的结构参数列于表 1 , 并将原子间的 Mulliken 集居数列于表 2. 需要说 明的是, 表中涉及的原子有的并非来自形成复合物 的同一分子.

各个物种的分子振动频率的计算分析表明, 所 有的反应物和产物的振动频率全部为正值, 说明它 们均可以稳定存在; 而 4 个过渡态的振动频率则有
且只有一个虚振动频率 (在 B3LYP/6-31G (d) 水平 上, 计算得到的未经标度的虚振动波数分别为 i1878、i1607、i1290 和 i1382 $\mathrm{cm}^{-1}$ ), 表明它们分别是 各自势能超曲面上的一级鞍点, 而且虚振动模式矢 量 (图从略) 展示了从反应物 (或中间物) 走向产物 (或者另一个中间物)的趋势, 表明过渡态是真实可 信的. 计算还表明, 反应 $\mathrm{a} 、 \mathrm{c}$ 和 $\mathrm{d}$ 的各物种均保持 平面构型, 但若将反应 (b) 中的各物种初始结构保持 平面, 则优化后进行的振动分析结果将会出现一个 振动模式为水分子中羟基基团的面外弯曲振动虚 频, 因此水参与形成的复合物 (包括过渡态) 均不能 保持为平面构型。使用半经验方法 (PM3 和 AM1) 和从头算的方法 (B3LYP/SVP) 的计算结果也支持 这一结论 ${ }^{[17-18]}$. 
表 1 质子转移过程中各物种部分结构参数和氢键参数 (键长: pm; 键角: $\left({ }^{\circ}\right)$ )

Table 1 Optimized structural parameters of species and H-bond parameters for complexes (bond lengths in pm and angles in degree)

\begin{tabular}{|c|c|c|c|c|c|c|c|c|c|c|c|c|}
\hline & \multicolumn{3}{|c|}{$\mathrm{a}$} & \multicolumn{3}{|c|}{$\mathrm{b}$} & \multicolumn{3}{|c|}{$\mathrm{c}$} & \multicolumn{3}{|c|}{ d } \\
\hline & Hy & $\mathrm{TS} 1$ & Py & IM1 & $\mathrm{TS} 2$ & IM2 & IM3 & TS3 & IM4 & IM5 & $\mathrm{TS} 4$ & IM6 \\
\hline$R_{\mathrm{N}-\mathrm{C}}$ & 133.1 & 136.5 & 141.4 & 134.2 & 137.0 & 140.2 & 134.2 & 136.2 & 139.3 & 1. 343 & 136.5 & 139.3 \\
\hline$R_{\mathrm{C}-\mathrm{O}}$ & 135.5 & 129.0 & 122.7 & 133.9 & 129.4 & 124.4 & 133.0 & 129.4 & 124.9 & 132.8 & 128.5 & 124.7 \\
\hline$R_{\mathrm{O}-\mathrm{H}}$ & 97.5 & 137.7 & 244.4 & 99.6 & 121.7 & 184.0 & 100.9 & 119.5 & 175.3 & 100.4 & 120.8 & 167.4 \\
\hline$R_{\mathrm{N}-\mathrm{H}}$ & 223.9 & 130. 2 & 101.4 & 192.8 & 127.2 & 103.1 & 174.9 & 130.3 & 104.1 & 188.7 & 129.1 & 104.0 \\
\hline$\angle \mathrm{N}-\mathrm{C}-\mathrm{O}$ & 117.2 & 105.6 & 120.0 & 119.1 & 118.3 & 120.2 & 119.4 & 120.2 & 120.6. & 120.3 & 120.7 & 120.4 \\
\hline$\angle \mathrm{C}-\mathrm{O}-\mathrm{H}$ & 105.6 & 75. 1 & 56.8 & 108.9 & 105.6 & 108. 6 & 110.6 & 114.8 & 122.2 & 112.8 & 119.0 & 125.3 \\
\hline$\angle \mathrm{C}-\mathrm{N}-\mathrm{H}$ & 56.5 & 75.1 & 114.1 & 106.5 & 105.9 & 113.8 & 124.0 & 120.8 & 116.5 & 121.7 & 117.7 & 115.1 \\
\hline$R_{\mathrm{O}-\mathrm{H} \cdots \mathrm{N}}$ & & & & 278.0 & 242.9 & 280.4 & 275.3 & 249.6 & 279.3 & & & \\
\hline$R_{\mathrm{O} \cdots \mathrm{H}-\mathrm{N}}$ & & & & & & & 275.4 & 249.6 & 279.3 & & & \\
\hline$\angle \mathrm{O}-\mathrm{H}^{\cdots} \mathrm{N}$ & & & & 142.8 & 149.8 & 146.7 & 174.0 & 175.8 & 179.1 & & & \\
\hline$\angle \mathrm{O} \cdots \mathrm{H}-\mathrm{N}$ & & & & & & & 174.1 & 175.8 & 179.1 & & & \\
\hline$R_{\mathrm{O}-\mathrm{H} \cdots \mathrm{O}}$ & & & & 271.9 & 240.2 & 274.6 & & & & 266.9 & 241.1 & 266.9 \\
\hline$\angle \mathrm{O}-\mathrm{H}^{\cdots} \mathrm{O}$ & & & & 161.3 & 157.3 & 150.9 & & & & 170.1 & 172.4 & 170.1 \\
\hline$R_{\mathrm{N}-\mathrm{H} \cdots \mathrm{N}}$ & & & & & & & & & & 292.4 & 258. 2 & 292.4 \\
\hline$\angle \mathrm{N}-\mathrm{H} \cdots \mathrm{N}$ & & & & & & & & & & 174.3 & 177.8 & 174.3 \\
\hline
\end{tabular}

Nimlos 等人 ${ }^{[19]}$ 曾经根据飞行时间质谱实验提 出 Py 为一非平面结构, 但随后的微波谱实验不能支 持这一推断 ${ }^{[20]}$, 理论计算表明平面结构的 Hy 和 Py 均对应于真正的能量极小点 ${ }^{[21]}$, 本文的计算同样表 明平面构型的 Hy 和 Py 也是稳定的, 在振动分析中 没有虚振动频率出现。

表 2 给出了从反应物到过渡态, 再到产物的过 程中, 参加质子转移的关键原子间 Mulliken 集居的 变化情况. 可以看到, $\mathrm{N}-\mathrm{H}$ 从作用较弱到形成化 学键的趋势, 而 $\mathrm{O}-\mathrm{H}$ 则朝着相反的趋势变化. 同 时 $\mathrm{C}-\mathrm{N}$ 键强度有所减弱 (键长有所缩短), $\mathrm{C}-\mathrm{O}$ 则 从单键变为双键。
在 $\mathrm{Hy}$ 和 $\mathrm{Py}$ 的结构互变过程中, 4 种反应途径 均形成了一个含有 $N 、 C 、 O 、 H$ 的多元环. 其中, 直 接的分子内质子转移过程 $\mathrm{a}$ 中, 由 $\mathrm{Hy}$ 提供的原子 形成了四元环, 在水助催化的反应过程中, 则还有水 分子中的 $\mathrm{H}$ 和 $\mathrm{O}$ 原子参与形成了一个六元环, 而在 双分子二聚体质子转移的反应 $\mathrm{c}$ 和 $\mathrm{d}$ 中, 则分别形 成了八元环。后 3 种反应途径复合物的一个显著特 点是在反应中有氢键形成。在反应中, 一共有 3 种 类型的氢键出现, 分别为 $\mathrm{O}-\mathrm{H} \cdots \mathrm{N}(\mathrm{N}-\mathrm{H} \cdots \mathrm{O})$, $\mathrm{O}-\mathrm{H} \cdots \mathrm{O}$ 和 $\mathrm{N}-\mathrm{H} \cdots \mathrm{N}$, 在每个复合物分子中均含 有 2 个氢键, 表 1 的下半部分列出了这些氢键的键 长和键角计算值. 一般来说, 键长越短, 键角越接近

表 2 部分原子间的 Mulliken 集居数变化(B3LYP/6-31G (d))

Table 2 The change of Mulliken population between key atoms in the proton transfer processes

\begin{tabular}{|c|c|c|c|c|c|c|c|c|c|c|c|c|}
\hline & \multicolumn{3}{|c|}{$\mathrm{a}$} & \multicolumn{3}{|c|}{$\mathrm{b}$} & \multicolumn{3}{|c|}{ c } & \multicolumn{3}{|c|}{ d } \\
\hline & Hy & $\mathrm{TS} 1$ & Py & IM1 & $\mathrm{TS} 2$ & IM2 & IM3 & TS3 & IM4 & IM5 & TS4 & IM6 \\
\hline $\mathrm{H}-\mathrm{N}$ & 0.014 & 0. 104 & 0.281 & 0.069 & 0.113 & 0.227 & 0.099 & 0.118 & 0.187 & 0.100 & 0.130 & 0. 194 \\
\hline $\mathrm{N}-\mathrm{C}$ & 0.448 & 0.308 & 0.205 & 0.394 & 0.299 & 0.239 & 0.403 & 0.335 & 0.263 & 0.404 & 0.321 & 0.267 \\
\hline $\mathrm{C}-\mathrm{O}$ & 0.293 & 0. 390 & 0.596 & 0.329 & 0.363 & 0.504 & 0.344 & 0. 367 & 0.482 & 0.349 & 0.373 & 0.477 \\
\hline $\mathrm{O}-\mathrm{H}$ & 0.229 & 0.146 & 0.011 & 0. 194 & 0.127 & 0.068 & 0.172 & 0.141 & 0.099 & 0.173 & 0.128 & 0. 089 \\
\hline
\end{tabular}


表 3 质子转移过程中各物种的能量和零点振动能 (ZPVE) (a. u. )

Table 3 Calculated energies and scaled zero-point vibrational energies (a. u) of species in four processes

\begin{tabular}{|c|c|c|c|c|c|c|c|c|}
\hline & \multicolumn{2}{|c|}{$\mathrm{a}$} & \multicolumn{2}{|c|}{$\mathrm{b}$} & \multicolumn{2}{|c|}{$\mathrm{c}$} & \multicolumn{2}{|c|}{$\mathrm{d}$} \\
\hline & $E_{\mathrm{RB}+\mathrm{HF}-\mathrm{LYP}}$ & ZPVE & $E_{\mathrm{RB}+\mathrm{HF}-\mathrm{LYP}}$ & ZPVE & $E_{\mathrm{RB}+\mathrm{HF}-\mathrm{LYP}}$ & ZPVE & $E_{\mathrm{RB}+\mathrm{HF}-\mathrm{LYP}}$ & ZPVE \\
\hline $\mathrm{R}$ & -323.51589 & 0.09184 & -399.94865 & 0.11701 & -647.06168 & 0. 18497 & -647.06365 & 0. 18548 \\
\hline TS & -323.45870 & 0.08693 & -399.92784 & 0.11095 & -647.05310 & 0. 17738 & -647.04855 & 0. 17715 \\
\hline $\mathrm{P}$ & -323.51791 & 0.09206 & -399.95196 & 0.11717 & -647.07213 & 0. 18610 & -647.06365 & 0. 18548 \\
\hline
\end{tabular}

$180^{\circ}$, 则氢键强度越大. 比较各个反应物和产物的 氢键参数. 对于反应 $b, O-H \cdots N$ 和 $\mathrm{O}-\mathrm{H} \cdots \mathrm{O}$ 的 键长要略高于在反应 $\mathrm{c}$ 和 $\mathrm{d}$ 的相应值. 更重要的 是, 与其它 2 个反应中的氢键键角接近直线 $\left(170.1^{\circ}\right.$ $\left.\sim 179.1^{\circ}\right)$ 相比, 键角要小得多 $(2$ 个氢键键角分别 为 $142.8^{\circ} \sim 161.3^{\circ}$ ), 因此反应 $\mathrm{b}$ 的氢键强度明显弱 于其他 2 个反应。在反应 $\mathrm{c}$ 和 $\mathrm{d}$ 中, 氢键强度则要 大得多, 例如在 $\mathrm{Hy} \cdots \mathrm{Py}$ 复合物中, $\mathrm{O}-\mathrm{H} \cdots \mathrm{O}$ 和 $\mathrm{N}-\mathrm{H} \cdots \mathrm{N}$ 的键角分别为 $170.1^{\circ}$ 和 $174.3^{\circ}$, 接近理 想的直线, 而键长则分别为 266.9 和 $292.4 \mathrm{pm}$, 均 小于冰中和 $\mathrm{NH}_{3}$ 中的相应键长 (分别为 276 和 338 $\mathrm{pm})^{[21]}$.

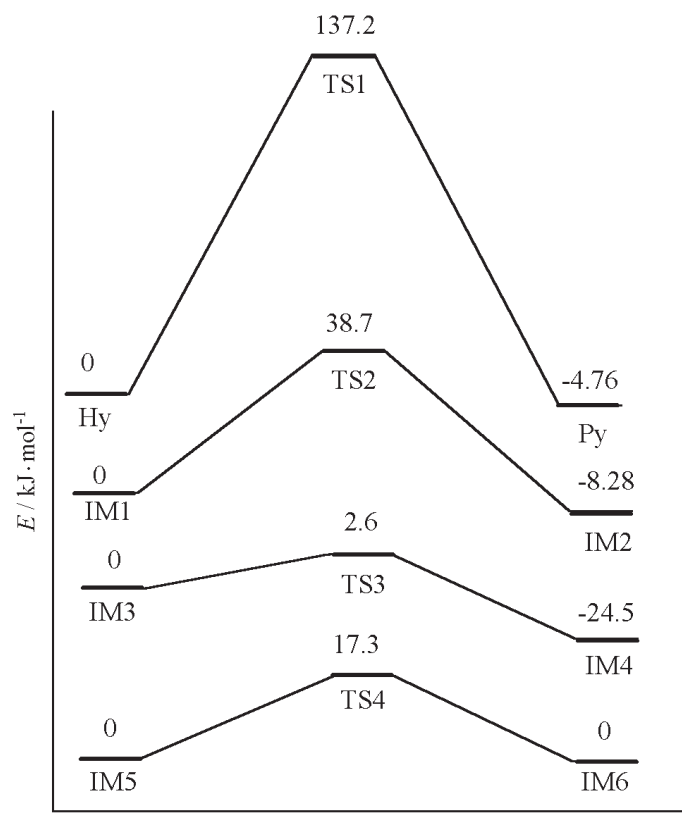

图 3 反应途径能量变化示意图 $\left(\mathrm{kJ} \cdot \mathrm{mol}^{-1}\right.$, 反应物能量作 为零点)

Fig. 3 Schematic diagram of potential energy surfaces describing intra- and intermolecular proton transfer (in $\mathrm{kJ} \cdot \mathbf{m o l}^{-1}$, the energies of reactants were taken as zero respectively )
表 3 列出了计算得到的各物种的能量和零点振 动能 (ZVPE)。根据文献 [23], 对 ZVPE 进行了标度 (标度因子 0.9804 ). 图 3 则给出了 4 种反应过程中 的总能量变化.

不同的实验方法,包括 UV/IR, XRD, XPS, 气 相 IR 实验和微波谱均认为 $\mathrm{Hy}$ 要比 $\mathrm{Py}$ 稳定 ${ }^{[24-25]}$, 它们之间的能量差异为 $2 \sim 3 \mathrm{~kJ} \cdot \mathrm{mol}^{-1}$, 实验测定的 反应的平衡常数 $K_{\mathrm{T}}(\mathrm{Py} / \mathrm{Hy})$ 为 $0.36 \sim 0.5$ (温度范 围在 $308 \sim 405 \mathrm{~K}$ ). 本文的计算结果则表明 Py 更稳 定, 其能量要比 $\mathrm{Hy}$ 低 $4.8 \mathrm{~kJ} \cdot \mathrm{mol}^{-1}$. 实际上, 由于 二者的能量差别是如此之小, 很多采用 Hartree-Fock 方法的 $a b$ initio 和密度泛函理论方法计算得到的结 果给出了一些矛盾的结果, 即使是高级别的包含电 子相关的从头算给出的结果范围也在 $-14.5 \sim+$ $1.56 \mathrm{~kJ} \cdot \mathrm{mol}^{-1}$ (正号表示 Py 更稳定) ${ }^{[26-27]}$. 例如, 采用 MP4(fc) [SDTQ]/DZ(d, p)//MP2(full)/ 6-31G(d) 方法, 计算得到的结果, 仍然为 Py 更稳定 (总能量比 $\mathrm{Hy}$ 低 $0.64 \mathrm{~kJ} \cdot \mathrm{mol}^{-1}$ ) ${ }^{[28]}$. 总的来说, 实 验和理论计算结果都显示二者的能量差别很小, 表 明在通常情况下都可以稳定存在 .

通过解离能的计算可以考察复合物形成前后的 能量变化情况. 这里解离能定义为复合物与其 2 个 单体间的能量差的负值. 不考虑零点振动能的校 正, 在 B3LYP/6-31G(d) 水平上, $\mathrm{Hy} \cdots \mathrm{H}_{2} \mathrm{O}$ 和 $\mathrm{Py} \cdots$ $\mathrm{H}_{2} \mathrm{O}$ 的解离能分别为 62.5 和 $65.9 \mathrm{~kJ} \cdot \mathrm{mol}^{-1}$, 而 $\mathrm{Hy} \cdots \mathrm{Hy} 、 \mathrm{Py} \cdots \mathrm{Py}$ 和 $\mathrm{Hy} \cdots \mathrm{Py}$ 的解离能分别为 78.5 、 95.3 和 $78.2 \mathrm{~kJ} \cdot \mathrm{mol}^{-1}$ (复合物). 在后 3 种复合物 中, Py $\cdots \mathrm{Py}$ 的解离能最大, $\mathrm{Hy} \cdots \mathrm{Hy}$ 和 $\mathrm{Hy} \cdots \mathrm{Py}$ 的解 离能则相差不大 ${ }^{[5]}$. 与无差基的吡啶能够与水混溶 不同, 2- 差基吡啶在水中的饱和溶解质量分数为 $0.5^{[29]}$, 即 2 - 差基吡啶倾向于互相结合, 这里的计算 结果与实验一致。

从图 3 反应过程中的能量变化可以看到, 进行 分子内的直接质子转移的反应 $\mathrm{a}$ 所需要的活化能最 
高, 达到 $137.2 \mathrm{~kJ} \cdot \mathrm{mol}^{-1}$. 由于具有如此高的反应 活化能, 因此在通常温度下, 这个反应很难进行. 分 子内质子转移过程需要很高的活化能, 主要原因可 能是在形成过渡态的过程中, 4 个关键原子形成的 四元环具有很大的张力而造成的, 在 Hy 或 Py 中, $\mathrm{C} 、 \mathrm{~N}$ 和 $\mathrm{O}$ 一般采取 $s p^{2}$ 或 $s p^{3}$ 杂化形式, 其理想的 键角应当分别在 $109.5^{\circ}$ 和 $120^{\circ}$, 但在 TS1 中, C $\mathrm{O}-\mathrm{H}$ 和 $\mathrm{C}-\mathrm{N}-\mathrm{H}$ 的键角分别只有 $75.1^{\circ}$. 相比之 下, 在后 3 种反应历程中, 由于形成的是六元环或者 八元环,共价键受到的张力要小得多.

当水作为催化剂参与反应后, 所需要的活化能 则大幅度降低, 只需要 $38.7 \mathrm{~kJ} \cdot \mathrm{mol}^{-1}$. 这 2 个反应 的活化能计算结果与文献 ${ }^{[18]}$ 报导十分接近 (在 $\mathrm{MP} 2 / \mathrm{SVP}$ 水平上计算的活化能分别为 146.4 和 $52.3 \mathrm{~kJ} \cdot \mathrm{mol}^{-1}$ ). 同时在文献中还提出了 1 种有 2 个水分子参与的质子转移过程的反应机理, 但是其 活化能比只有一个水分子参与的反应活化能还要高 约 $4 \mathrm{~kJ} \cdot \mathrm{mol}^{-1}$. 这里还可以看到, $\mathrm{Py} \cdots \mathrm{H}_{2} \mathrm{O}$ 的总能 量比 $\mathrm{Hy} \cdots \mathrm{H}_{2} \mathrm{O}$ 低 $8.28 \mathrm{~kJ} \cdot \mathrm{mol}^{-1}$, 表明体系更倾向 于形成 Py 与水的复合物, 实验也表明, 在处于固体 状态和高介电常数的极性溶剂中 (例如水作为溶剂 时), 平衡将向生成 Py 的方向移动, 即 Py 将变得比 Hy 稳定 ${ }^{[30]}$.

与前 2 种反应机理相比, 后 2 种反应历程所需 要的活化能更低. 在反应 $\mathrm{c}$ 中, 过渡态 TS3 的总能 量仅比反应物高 $2.6 \mathrm{~kJ} \cdot \mathrm{mol}^{-1}$ (复合物), 因此 $\mathrm{Hy}{ }^{\cdots} \mathrm{Hy}$ 可以很容易越过这个能垒, 而逆反应则有 $27.1 \mathrm{~kJ} \cdot \mathrm{mol}^{-1}$ (复合物), 表明 $\mathrm{Py} \cdots \mathrm{Py}$ 要转变为醇 式复合物则困难得多. 而在反应历程 $\mathrm{d}$ 中, 反应能 垒也比反应 $\mathrm{b}$ 低很多, 正逆反应的活化能都只有 $17.3 \mathrm{~kJ} \cdot \mathrm{mol}^{-1}$ (复合物), 当然这种质子转移不会 改变体系中的 Hy 和 Py 浓度, 但它仍然是一个可能 发生的基元反应. 比较 2 个反应的逆反应, 可以发 现逆反应可能以反应 $\mathrm{d}$ 的方式进行更有利。总的来 说, 这 2 种反应途径都要比水催化反应途径更容易 进行。同时, 这种类似于自催化的反应也更加合理 . 至于后 2 种反应机理有更低的反应活化能, 与前 面分析的氢键强度要大于水分子与 Hy 或者 Py 形 成的氢键强度, 同时受到的张力较小有很大关系.

\section{3 结 论}

(1)计算得到的 2-差基吡啶的醇式结构和酮式 结构的能量差别很小, 表明在通常情况下, 二者均可 以稳定存在时。与水结合, Py 能量降低更多.

(2)通过对图 2 所示的 4 种可能的反应机理的 研究表明, 直接进行的分子内质子转移过程需要最 大的活化能 (137. $\left.2 \mathrm{~kJ} \cdot \mathrm{mol}^{-1}\right)$; 当水作为催化剂时, 反应活化能降低到 $38.7 \mathrm{~kJ} \cdot \mathrm{mol}^{-1}$; 形成 $\mathrm{Hy} \cdots \mathrm{Py}$ 复 合物后, 活化能进一步降低到只有 $2.6 \mathrm{~kJ} \cdot \mathrm{mol}^{-1}$, 反应极其容易进行, 此时逆反应的活化能为 27.1 $\mathrm{kJ} \cdot \mathrm{mol}^{-1}$ (复合物); 在形成 $\mathrm{Hy} \cdots \mathrm{Hy}$ 复合物后, 正 逆反应的活化能均为 $17.3 \mathrm{~kJ} \cdot \mathrm{mol}^{-1}$ (复合物).

(3) 在反应过程中, 氢键起着非常重要的作用, 它的形成大幅度降低了反应的活化能, 在 Hy 和 Py 之间形成的氢键强度要大于水与 Hy 或者 Py 形成 的氢键, 同时张力也是一个重要的影响因素.

\section{References}

1 Kwiatkowski J S, Barlett R J, Person W B. J. Am. Chem. Soc., 1988, 110: 2353

2 Yen S J, Ho J J. J. Phys. Chem. A, 2000, 104: 8551

3 Nguyen K A, Gordon M S, Truhlar D G. J. Am. Chem. Soc., 1991, 113: 1596

4 Kong DX, Chen DZ, Zhang HY. Chinese J. Struct. Chem. 2000, 19 (6): 449 [孔德信, 陈德展, 张红雨. 结构化学 (Jiegou Huaxue), 2000, 19 (6): 449]

5 Chou P T, Wei C Y. J. Phys. Chem. B, 1997, 101: 9119

6 Leach A R. Molecular Modelling: the Principles and Applications. London: Addison Wesley Longman Press, 1996: 194

7 Tian S X, Zhang C F, Zhang Z J, Chen X J, Xu K Z. Chem. Phys., 1999, 242: 217

8 Gorb L, Leszczynski J. J. Am. Chem. Soc., 1998, 120: 5024

9 Person W B, Szczepaniak K, Szczesniak M, Kwiatkowski J S, Hermandez L, Czeminski R. J. Mol. Struct., 1989, 194: 239

10 Li YH, Hong SG, Feng WL, Lei M. Acta Phys. -Chim. Sin., 2000, 16(11): 992 [李永红, 洪三国, 冯文林, 雷 鸣. 物理化学学报 (Wuli Huaxue Xuebao), 2000, 16(11): 992]

11 Hou RB, Chen ZD, Yi XH, Bian J, Xu GX. Sci. in China (Ser. B), 2001, 31(1):6 [侯若冰, 陈志达, 义祥辉, 市江, 徐光宪. 中国科学 (B 辑), 2001,31(1): 6]

12 Krebs C, Hoffman H J, Kohler H J, Weiss C. Chem. Phys. Lett., 1980, 69: 537

13 Lee C, Yang W, Parr RG. Phys. Rev. B, 1988, 37: 785

14 Becke A D. J. Chem. Phys., 1993, 98: 5648

15 Schlegel H B. J. Comp. Chem., 1982, 3: 214

16 Frisch M J, Trucks G W, Schlegel H B, Scuseria G E, Robb MA, Cheeseman J R, Zakrzewski V G, Montgomery J A, Stratmann Jr 
R E, Burant J C, Dapprich S, Millam J M, Daniels A D, Kudin K N, Strain M C, Farkas O, Tomasi J, Barone V, Cossi M, Cammi R, Mennucci B, Pomelli C, Adamo C, Clifford S, Ochterski J, Petersson G A, Ayala P Y, Cui Q, Morokuma K, Malick D K, Rabuck A D, Raghavachari K, Foresman J B, Cioslowski J, Ortiz J V, Stefanov B B, Liu G, Liashenko A, Piskorz P, Komaromi I, Gomperts R, Martin R L, Fox D J, Keith T, Al-Laham M A, Peng C Y, Nanayakkara A, Gonzalez C, Challacombe M, Gill P M W, Johnson B, Chen W, Wong M W, Andres J L, Gonzalez C, Head-Gordon M, Replogle E S, Pople, J. A. Gaussian 98, Pittsburgh PA: Gaussian, Inc., 1998

17 Morpurgo S, Bossa M, Morpurgo G O. J. Mol. Struct. ( Theochem), 1998, 429: 71

18 Barone V, Adamo C. J. Phys. Chem., 1995, 99: 15062

19 Nimlos M R, Kelley D F, Bermstein E R. J. Phys. Chem., 1989, 93: 643

20 Hatherley L D, Brown R D, Godfrey P D, Pierlot A P, Caminati W, Damiani D, Melandri S, Favero L B. J. Phys. Chem., 1993, 97: 46

21 Wong M W, Wiberg KB, Frish M J. J. Am. Chem. Soc., 1992,

114: 1645

22

23

25 Nowak M J, Lapinski L, Fulara J, Les A, Adamowicz L. J. Phys. Chem., 1992, 96: 1562

26 Dkhissi A, Houben L, Smets J, Adamowicz L, Maes G. J. Mol. Struct., 1999, 484: 215

27 Matyus P, Fuji K, Tanaka K. Tetrahedron, 1994, 50: 2405

28 Kwiatkowski J S, Leszcynski J. J Mol. Struct. (Theochem), 1994, 312: 312

29 Yao ZP, Li QG, Dong TW. Chin. Univ. Chem., 1993, 8 (4): 23 [姚子鹏, 李全根, 董庭威. 大学化学 (Daxue Huaxue), 1993, 8 (4): 23]

30 Kuzuya M, Noguchi A, Okuda T. J. Chem. Soc., Perkin Trans. 2, 1985: 1423

\title{
A Theoretical Study on the Proton Transfer in 2-hydroxy Pyridine*
}

\author{
Chen Wen-Kai Xu Jiao Zhang Yong-Fan Zhou Li-Xin Li Jun-Qian \\ ( Department of Chemistry, Fuzhou University, State Key Laboratory of Structural Chemistry, Fuzhou 350002 )
}

\begin{abstract}
Abstrat The processes of the proton transfer between 2-hydroxy pyridine (Hy) and 2(1H) -pyridone (Py) were investigated by using density functional theory of quantum chemistry method at B3LYP/6-31G(d) level. The four possible reaction pathways: (a) intramolecular proton transfer; (b) water-assisted intermolecular transfer; (c) isomerization by double-proton transfer in the dimer and (d) intermolecular proton transfer via the complex formed by Hy and Py were investigated in the present paper. The calculated results showed that the processes in (b), (c) and (d) has lower activation energies than that of the intramolecular tautomeric oxo-hydroxy reaction (a) because of the formation of hydrogen-bonded complexes in (b), (c) and (d). The activation energies corrected by scaled zero-point vibrational energies for the four reaction processes calculated at the B3LYP/6-31G (d) level are 137. 2, 38. 7, 2.6 and $17.3 \mathrm{~kJ} \cdot \mathrm{mol}^{-1}$, respectively. It is likely that the hydrogen bonds formed in the complexes play an important role in proton transfer processes in the later three reaction pathways.
\end{abstract}

Keywords: 2-hydroxy pyridine, Density functional theory, Quantum chemistry, Tautomerism, Activation energy 